\title{
Uncertain viscoelastic models with fractional order: a new spectral tau method to study the numerical simulations of the solution
}

\begin{abstract}
The analysis of the behaviors of physical phenomena is important to discover significant features of the character and the structure of mathematical models. Frequently the unknown parameters involve in the models are assumed to be unvarying over time. In reality, some of them are uncertain and implicitly depend on several factors. In this study, to consider such uncertainty in variables of the models, they are characterized based on the fuzzy notion. We propose here a new model based on fractional calculus to deal with the Kelvin-Voigt (KV) equation and non-Newtonian fluid behavior model with fuzzy parameters. A new and accurate numerical algorithm using a spectral tau technique based on the generalized fractional Legendre polynomials (GFLPs) is developed to solve those problems under uncertainty. Numerical simulations are carried out and the analysis of the results highlights the significant features of the new technique in comparison with the previous findings. A detailed error analysis is also carried out and discussed.
\end{abstract}

Keyword: Fractional differential equations (FDEs); Spectral methods; Legendre polynomials; Viscoelastic models 